\title{
Contribution to the Development of a Dynamic Circulation Map using the Multi-Agent Approach
}

\author{
Asmaa ROUDANE*, Mohamed YOUSSFI, Khalifa MANSOURI \\ Lab. SSDIA, ENSET, University Hassan II Casablanca, Morocco \\ Bd. HassanII, Mohammedia, 28820 Morocco
}

\begin{abstract}
Road traffic is considered one of the most difficult domains to manage and one of the fast- growing networks. This environment is geographically distributed and its actors are in continuous interaction in order to be able to succeed in a realtime exchange of the variable data of the road traffic. The modeling of this complex system requires powerful tools to be able to realize precise representations of reality hence the combination of mathematical modeling tools and intelligent systems. This paper presents a model of road traffic using graphs for the representation of the road infrastructure and the multiagent technology for the management of the data representing the information circulating in the road network. This new approach aims at optimizing traffic paths, minimizing the time of a given journey and developing a dynamic traffic map that will enable the user to reach his destination by following the best possible path.
\end{abstract}

Keywords-Road traffic; road traffic management systems; intelligent systems; complex systems; multi-agent systems; graphs; real-time

\section{INTRODUCTION}

New technologies such as artificial intelligence, collective intelligence, multi-agents technology, expert systems and realtime systems are used to facilitate the management of complex environments and to improve their functioning [1]. The study, modeling, and processing of complex systems by methods of collective intelligence apply to various fields of application such as population flow modeling [2], epidemiology, energy flow, social networks, and adaptive load distribution. Collective Intelligence systems are naturally complex and [3] have the ability to adapt to uncertain and unknown environments, [4] can organize themselves autonomously, and [5] present 'emergent' behavior. The complex environment in this contribution is road traffic.

The objectives of most road traffic studies are to improve the fluidity of traffic in the network by preventing and avoiding abnormal traffic events (saturation, congestion [6], etc.), and minimizing the travel time of each user by enabling him to reach his destination under the best possible conditions [7]. The achievement of these objectives depends first and foremost on the infrastructure of the road network, which must be expanded to accommodate new users, who are increasingly numerous and who share the same network. Since the existing road infrastructure is insufficient and its enlargement is not always so obvious, another solution is to improve road traffic management methods by sharing the same network.
Improving road traffic management requires the use of new technologies, in particular, collective intelligence. As already mentioned, road traffic is a very complex, geographically distributed environment and its components are in continuous interaction, for all these reasons, and for others the multi-agent approach is the most suitable for better road traffic management.

An agent-based approach makes it possible to design noncentralized and self-organizing management models that improve the responsiveness of decision-making systems, which increases their autonomy. The term "agent" denotes hardware or software that supports an autonomous decisionmaking context. Agents are not simply objects or actors but also autonomous entities trying to achieve goals by acting on their environment. The agents must have the following properties: autonomy, proactivity, adaptability, sociability and mobility [8]. Multi-agent technology facilitates the management of geographically distributed environments, reduces their complexity and ensures continuous interaction between their different actors [9].

The combination of the multi-agent approach with the modeling tools gives powerful models that help to effectively solve many problems. One of the most widely used modeling tools is the graphs.

This contribution firstly presents the first proposed solution which was the result of a comparative study of existing solutions for the management of road traffic. This solution has limitations which will be detailed in the problematic part; these limitations led us to think of another solution by combining the modeling of the road network using the graphs and the management of the data circulating in this network using multi-agent technology. This modeling will make it possible to optimize traffic flows, to minimize the travel time of a journey, and finally to produce a dynamic traffic map that reflects the real-time state of the road network.

\section{RELATED WORKS}

The complexity of road traffic makes its management difficult and costly. Effective management depends on the availability of traffic data that are variable all the time. In general, the problems that a road traffic management system must solve can be summarized as follows:

- Identification of the critical areas of the system being studied. 
- Detection of abnormal traffic events (saturation, congestion, accident ...).

- Rapid dissemination of information about abnormal events in order to keep a fluid state of traffic.

Several solutions have been proposed to meet the needs of road traffic managers. These solutions fall into two categories: tools to support real-time or deferred operations or decisionmaking tools. The tools for operating aid, or rather for regulation, are generally autonomous systems capable of performing an action in a given situation or under predefined conditions [10]. These systems make it possible to monitor the good progress of the operation and to monitor the quality of service offered to passengers. Decision support systems are solutions based on knowledge and collective intelligence; they collect information, analyze it, interpret it, symbolize it and finally present it to decision-makers in order to help them take the best decision for a given situation [11].

Most of the systems studied are based on multi-agent technology as there are others based essentially on the objectoriented approach. The comparative study considers two factors to present the applications studied. These two factors are:

The Coordination in each system by using three attributes:

- Control: centralized or distributed.

- Structure: static or dynamic.

- Attitude: cooperative or competitive, or both.

The Maturity or level of experience, considering the following four levels of experience:

a) Architectural proposal: a description of the idea and the principles with their characteristics.

b) Simulation: the tool has been validated in a simulation environment with real or simulated data.

c) Real environment: The tool has been validated in a real environment with limited or complete data.

d) Deployment: This is the highest level where the application has been implemented in the real world and has been used.

The comparative study is presented in Table 1:

TABLE I. COMPARATIVE STUdy OF EXISTING ROAD TRAFFIC MANAGEMENT SOLUTIONS

\begin{tabular}{|l|l|l|l|l|l|}
\hline System & Use & Structure & Coordination & Maturity & Type of data \\
\hline $\begin{array}{l}\text { TRYS (Work of } \\
\text { Cuena et al) [11] }\end{array}$ & DSS & MAS dynamic & $\begin{array}{l}\text { Distributed, } \\
\text { cooperative }\end{array}$ & Simulation & Artificial, limited \\
\hline InTRYS [12] & DSS & MAS static & $\begin{array}{l}\text { Centralized, } \\
\text { cooperative }\end{array}$ & Deployment & Real, limited \\
\hline TRYS A2 [12] & DSS & MAS static & $\begin{array}{l}\text { Distributed, } \\
\text { cooperative }\end{array}$ & Simulation & Real, complete \\
\hline $\begin{array}{l}\text { Work of Adler et al } \\
\text { [13] }\end{array}$ & DSS et OSS & MAS dynamic & $\begin{array}{l}\text { Distributed, } \\
\text { cooperative }\end{array}$ & Simulation & Artificial, complete \\
\hline $\begin{array}{l}\text { Work of Ossowski } \\
\text { et al [14] }\end{array}$ & DSS & MAS & $\begin{array}{l}\text { Distributed, } \\
\text { cooperative }\end{array}$ & Simulation & Artificial, limited \\
\hline $\begin{array}{l}\text { Work of Li et al } \\
\text { [15] }\end{array}$ & DSS & MAS static & $\begin{array}{l}\text { Distributed, } \\
\text { cooperative }\end{array}$ & Simulation & Artificial, limited \\
\hline $\begin{array}{l}\text { Work of Finder et } \\
\text { Strap [16] }\end{array}$ & DSS & Distributed Control & Distributed & $\begin{array}{l}\text { Proposition } \\
\text { architectural }\end{array}$ & - \\
\hline $\begin{array}{l}\text { Work of Van } \\
\text { Katwijk [17] }\end{array}$ & OSS & MAS & $\begin{array}{l}\text { Distributed, } \\
\text { cooperative }\end{array}$ & Simulation & Artificial, limited \\
\hline TRANSYT [18] & OSS differed & OOP & OOP (C++) & Deployment & Real, limited \\
\hline CLAIRE [19] & OSS real & OSS real & OOP & Deployment & Real, limited \\
\hline SCOOT [20] & Deployment & Real, limited \\
\hline
\end{tabular}

DSS: Decision Support System OSS: Operations Support System OOP: Object Oriented Programming MAS: Multi-Agents System

The solutions studied present some inputs in the management of road traffic but they are limited. These limitations are either in presenting time-delayed functionality, which is not efficient in the management of a variable environment, or in covering only one problem of road traffic. This comparative study has been an inspiration to design a first model of road traffic management based on multi-agent technology and the sharing of the studied area in sub-zones monitored by agents in continuous communication.

\section{First model:}

The idea of the model is to apply the two approaches: Distributed Artificial Intelligence (AI) and Multi-Agent approach. The AI approach is an approach for the control of large scale systems using decomposition and distribution. Large systems are decomposed into a series of small, interconnected subsystems, each responsible for controlling its domain and coordinating activities with adjacent subsystems. The AI approach creates a more robust environment in control side by allowing faster responses; sharing critical resources and increasing flexibility in adapting to changes in the system [21]. 
Each domain is monitored by a reactive agent whose mission is to collect road traffic data using equipment such as detectors or sensors and then transfer this data to a coordinating agent so that it can be sent to its turn to the area agent to be finally communicated to the central agent, as described in Figure 1. All these communications must be done in real time since the data collected are variable and unpredictable.

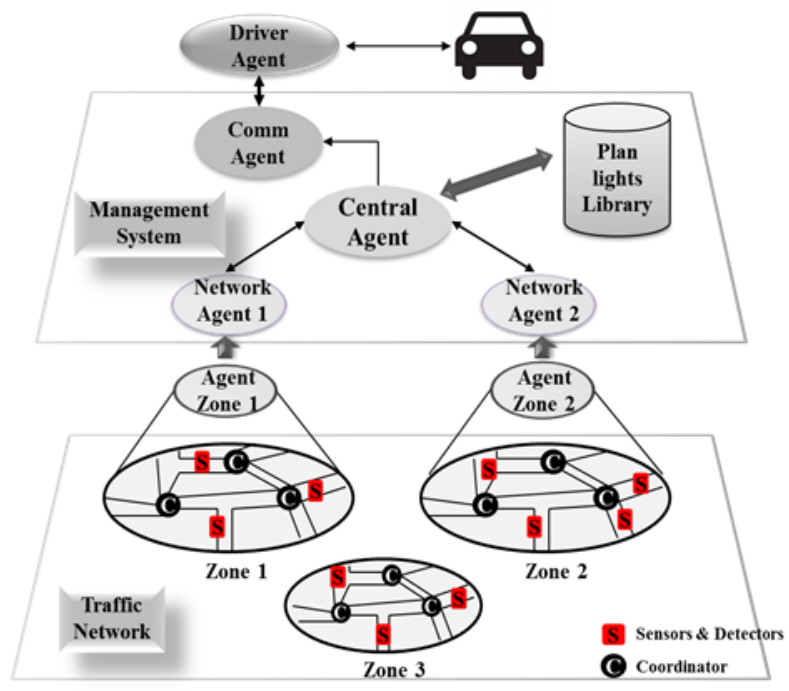

Fig. 1. First proposed model for road traffic management

The implementation of this model has shown that it is difficult to develop and succeed all communications between agents in real-time. In general, the modeling of complex systems, such as road traffic, by reactive agents uses a subsymbolic representation of the world through stimulus/action structures, that is, following a hierarchy of many simple automaton agents who need information on their local environment in order to decide on their actions [22]. This hierarchical architecture that has been adopted for agents is too complex to construct. To understand this difficulty, a communication diagram has been elaborated, in Figure 2, between agents.

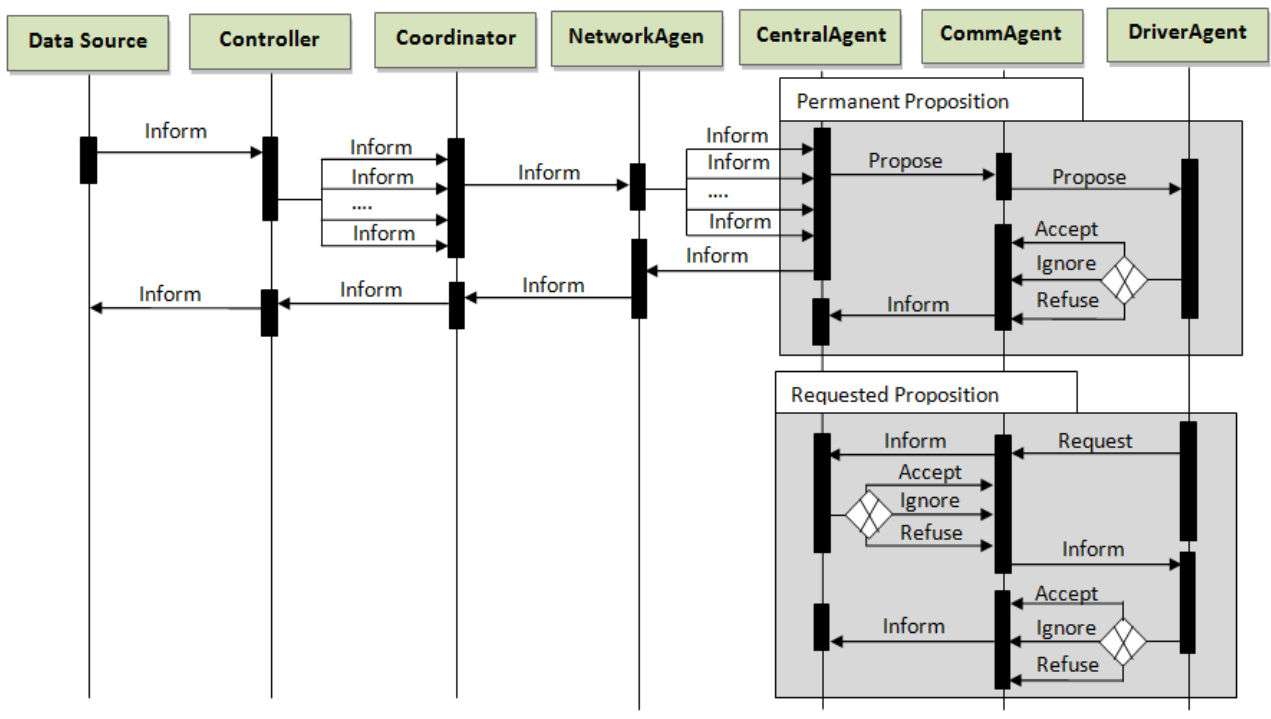

Fig. 2. Diagram of communications between the actors of the first proposed model for the management of road traffic

The analysis of the communications between the agents shows that there are several messages to be exchanged in real time, these messages contain variable data which must be communicated to the central agent before they change, which is not Always the case given the unpredictability of traffic data.
This analysis and other research have oriented the researches towards another idea in order to better manage road traffic. The new solution proposed is to separate the modeling of the infrastructure and the management of the data using one of the most powerful modeling tools: Graphs. 


\section{GRAPHS AND MULTI-AGENT SYSTEMS}

The field of application in this study is road traffic; this system depends on two components:

- Road infrastructure.

- Data circulating in the road network.

The proposed solution for road traffic management consists of modeling the road infrastructure using graphs and developing a traffic data tree that will be dynamically fed by agents using the multi-agent approach. The use of graphs is commonly current as a representation tool: the schematic map of streets, a genealogical tree, and the representation of a computer network; are examples of modeling using graphs. Graphs are standard objects of mathematical modeling; they have an expressive power to cover very wild fields of problems and applications [23].

A graph can be represented by several means, either by the description of the arcs, representing it by a list of summits, each being characterized by its name, a possible label, and the list of arcs that have this summit for origin. The representation can be sagittal that means in a drawing form, it can also be a matrix (adjacency matrix), or by a dictionary which consists of a table enumerating the following and preceding of each summit.

To represent a network or a complex system by a graph, it is first necessary to establish an analogy between the two. A network is a graph whose individuals and relationships have quantitative or qualitative characteristics. As for the complex system, it is a large number of interacting entities forming a set whose behavior is unpredictable for the observer because resulting from non-trivial interactions [24].

Then the entities of a complex system are associated to the summits of the graph, and the interactions between these entities are associated in turn with the graph arcs. However, graphs are discrete structures whose structure, topology is static, while complex systems are inherently dynamic and evolve over time, hence the separation of the infrastructure modeling, which is in general static, and data that are variable all the time and unpredictable.

\section{Modeling of road infrastructure:}

The complex system in the actual study is road traffic; Figure 3 presents a first modeling of the road network using the graphs:

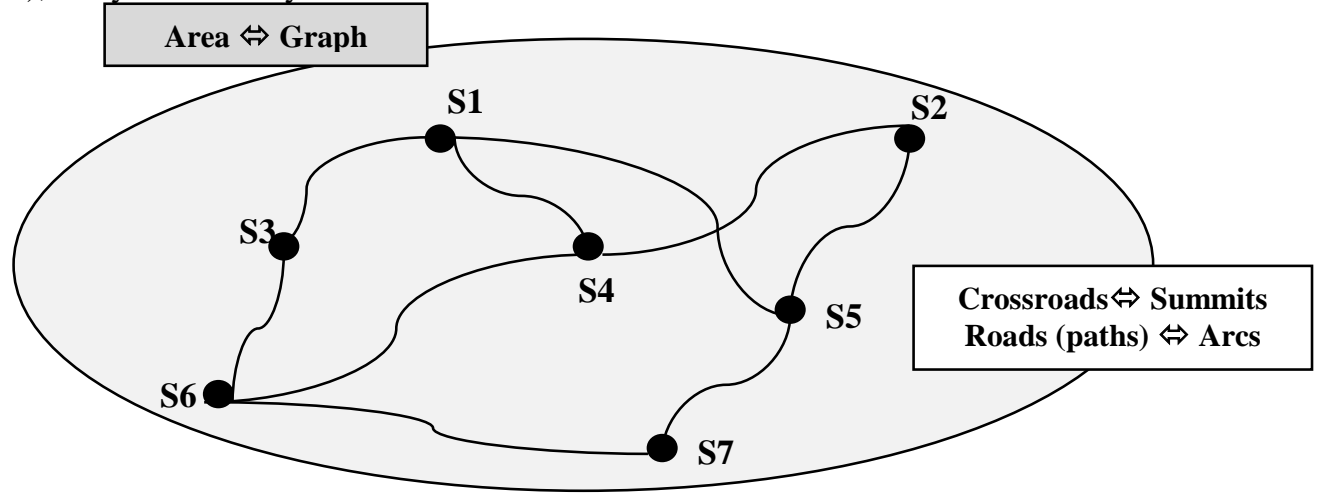

Fig. 3. Modeling the road infrastructure using graphs

Each area of the road network is considered as a graph whose summits are intersections or intersections, and the arcs are the roads or paths connecting each two crossroads. A zone is identified by a name, it contains several crossroads. A crossroads is identified by a name and its coordinates. A road connecting two crossroads is identified by a name, an origin and a destination, and the most important by a weight. In general; the weight of an arc is its value, in this case, the weight is the dynamic part of the data circulated in the network.

In this proposition the value of a road depends on several parameters, these parameters are either static or variable. Variable parameters are only the dynamic traffic data that is collected by the agents monitoring the road in question. Each crossroads has an agent who collects data concerning the roads connected to the crossroads in question, these data are issued by the mobile agents associated with the vehicles crossing the intersection. Then the agents of the crossroads are required to regularly transfer the collected information to the system agent whose mission is the updating of the dynamic traffic map which offers users the current state of the road network, as schematized in figure 4. 


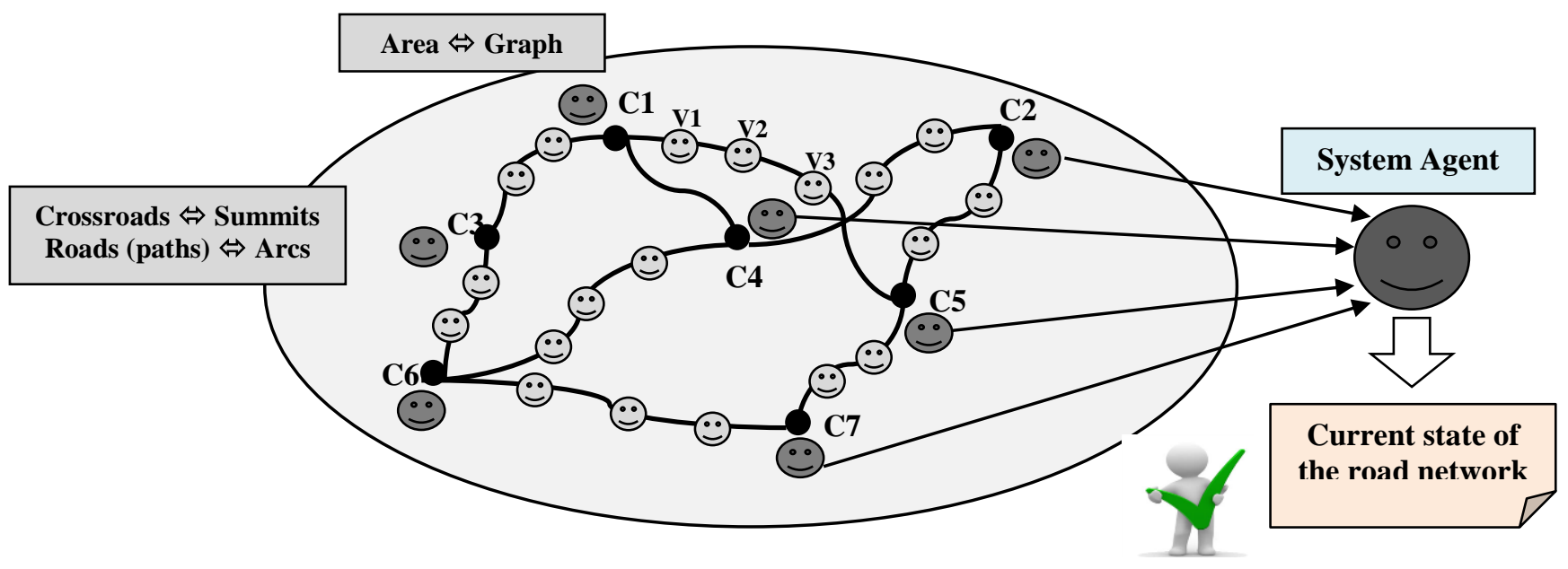

Fig. 4. Modeling of road traffic using graphs and the multi-agent approach

Each vehicle agent passing through a road is invited to send its current information to the agents of the nearby intersections. The latter transmits the received information to the system agent which analyzes the received messages in order to feed the traffic data tree to be able to update the dynamic traffic map to keep a real view of the road network. The interactions between the agents described above are schematized in the diagram presented in Figure 5.

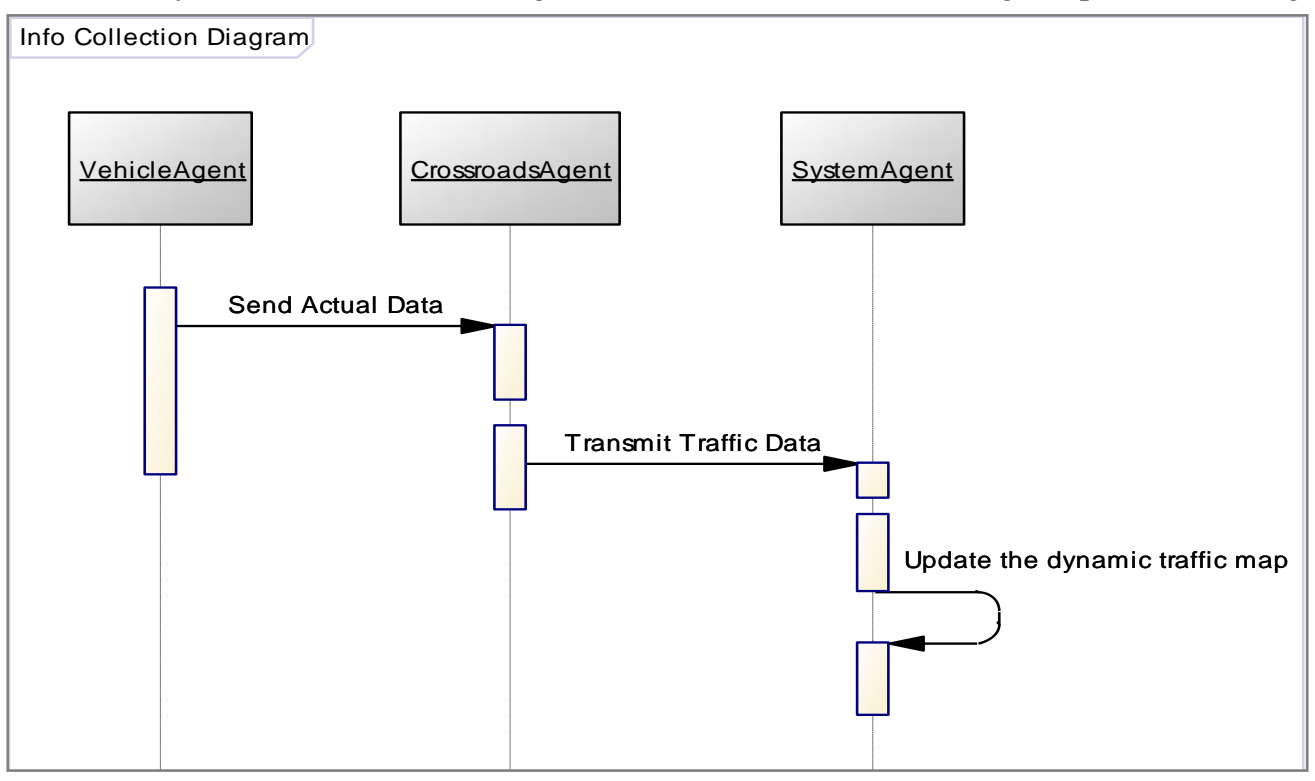

Fig. 5. Sequence diagram detailing road traffic data collection

For a user to reach his destination by browsing the best possible path he must connect to the system and indicate his destination, the system analyzes the information sent by the user and searches for all possible paths to the indicated destination. Thereafter, the system agent uses the optimization algorithms to choose the best possible path that is sent to the user afterward. All these exchanges are detailed in Figure 6. 


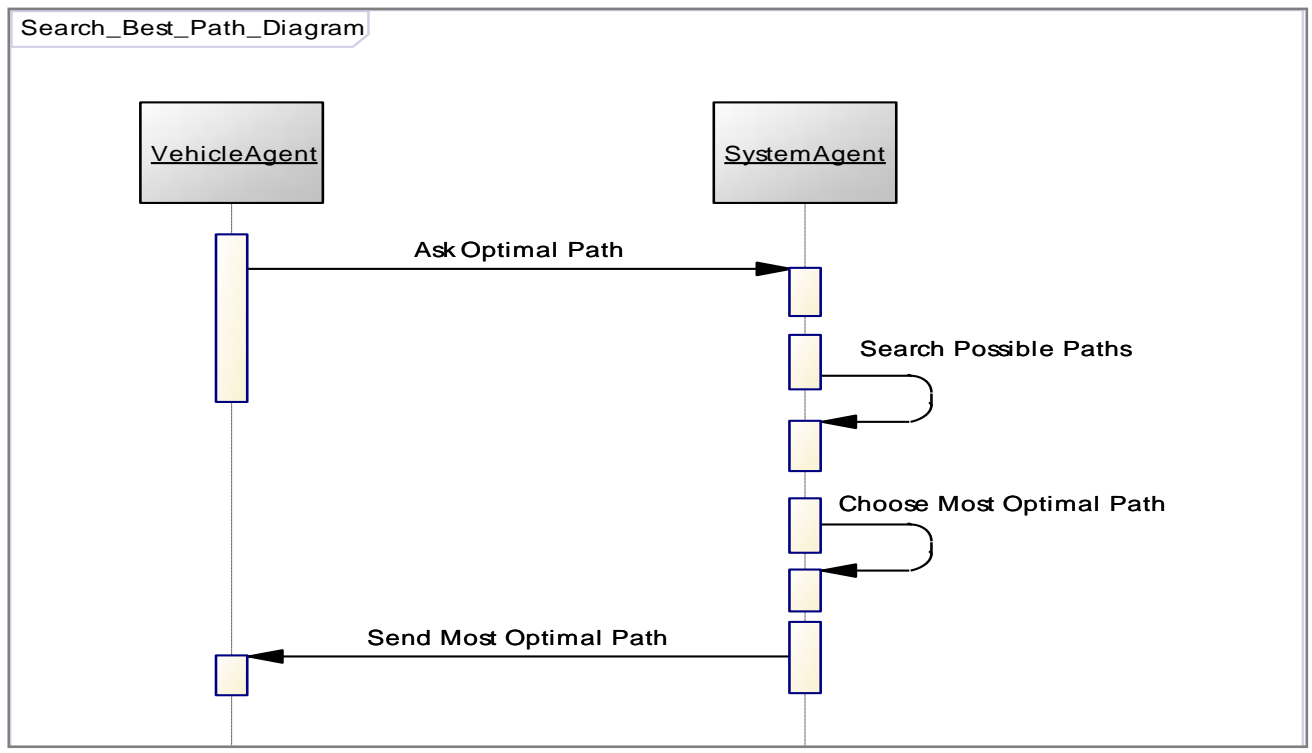

Fig. 6. Sequence diagram detailing the optimal path search

\section{The tree of the data circulating in the road network:}

As mentioned in the previous section, each road in the studied area is monitored by an agent. The mission of this agent is summarized in the collection of the variable data of the path in question and the transfer of this information to the system agent whose mission is to update the data tree described in Figure 7. The variable data (speed, actual distance ...) make it possible to evaluate each road in order to offer the user the best possible path to his destination.
The best possible path depends on several factors: it may be the shortest road (short distance), it can be the most secure road, or it can be a path that is distinguished by several characteristics. In the description of the data tree, a few parameters are presented which characterize each road; these parameters make it possible to improve the calculation of the value of each path as may be added to other information that may offer the user a better view of the best route to his destination. The parameters to be included in the assessment of the route must be well analyzed in order to give us an accurate classification of the paths to be covered.

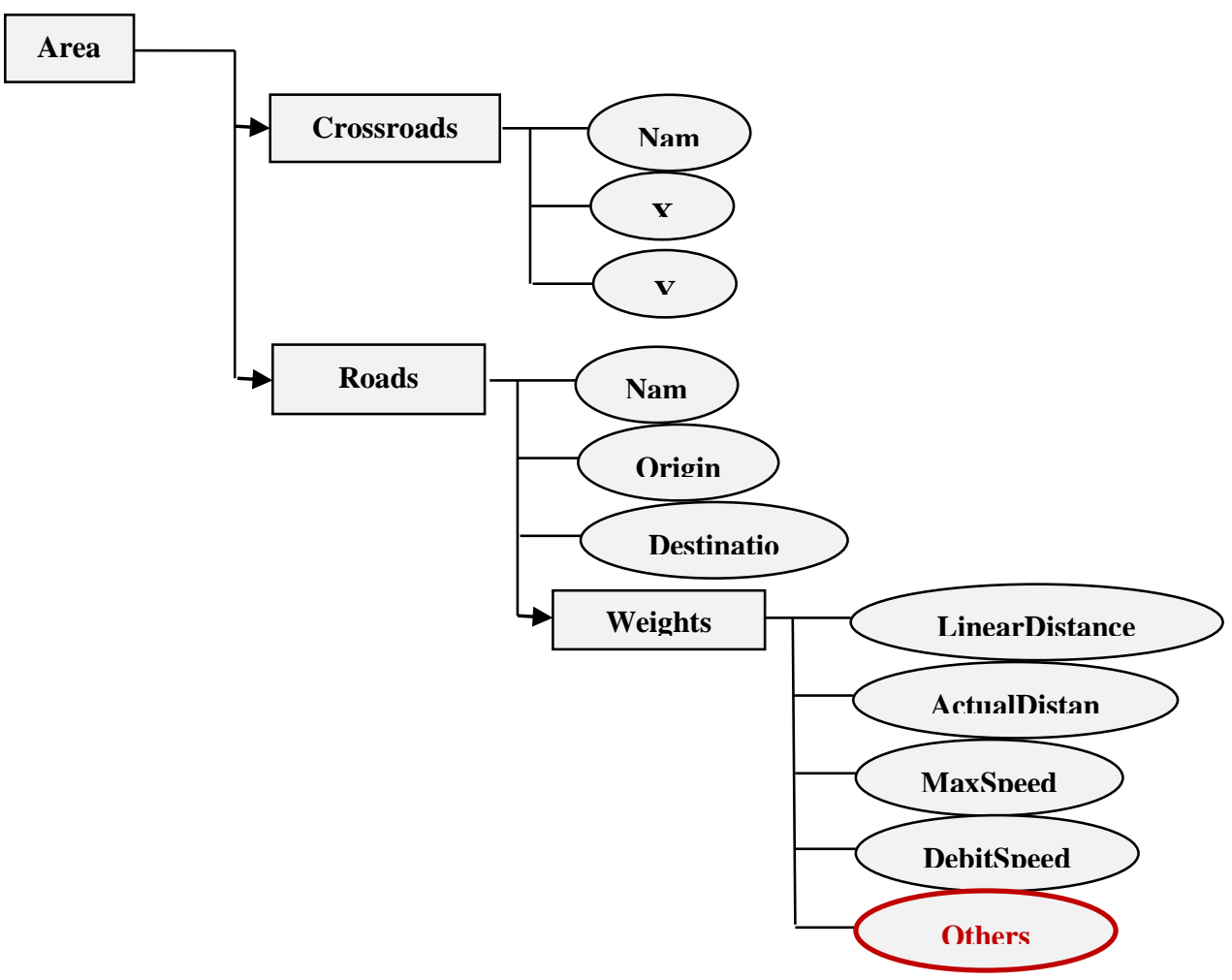

Fig. 7. The road traffic data tree 
The solution presented in this article has several objectives: one of them is the minimization of the journey time of a path. The travel time is an equation with two variables: the speed of flow and the actual distance, in other words, it is the relation between the distance actually traveled and the average speed with which this distance has been browsed. In this solution, the travel time is a function with several parameters that change in real time, and these parameters can be changed according to the preferences of the user.

The system agent in the proposed solution is an agent that has several missions:

- Update information in the road traffic data tree.

- Find the most optimal path to a specified destination using the optimization algorithms that will allow choosing the best route according to the state of the network and according to the user preferences.

Thus, by connecting to the proposed system, the user can have a real-time view of the state of the road network thanks to the dynamic traffic map offered by the system; in addition, he can indicate his preferences to be able to personalize the calculation of his travel time for the best possible path to his destination.

\section{CONCLUSION}

This paper presents a new modeling of road traffic based on graphs and multi-agent technology. This modeling consists in separating the representation of the road infrastructure using the specifications of the graphs and the representation of the data circulating in the road network using a data tree that will be managed in real time by agents based on the technology Multi-agents.

The new approach in this paper aims to optimize traffic routes, minimize travel time for a given destination, and develop a dynamic traffic map that will provide a real-time view of the current state of the road network to users; enabling them to reach their destinations by traveling the best path possible.

\section{REFERENCES}

[1] M. Mitchell, "Complex systems: Network thinking", Artif. Intell, vol. 170, no. 18, pp. 1194-1212, Dec. 2006.

[2] James M. Keller, Derong Liu, David B. Fogel, "Collective Intelligence and Other Extensions of Evolutionary Computation", Wiley-IEEE Press 1, pp. 400, 2016.

[3] P. Ball, Material witness: Designing with complexity, Nature Materials 3 (2004) 78

[4] A.-L. Barabási, Linked: The New Science of Networks, Perseus, New York, 2002.

[5] A.-L. Barabási, R. Albert, Emergence of scaling in random networks, Science 286 (2002) 509-512.
[6] Shen Wang, Soufiene Djahel, Zonghua Zhang, Jennifer McManis, "Next Road Rerouting: A Multiagent System for Mitigating Unexpected Urban Traffic Congestion", IEEE Transactions on Intelligent Transportation Systems, Vol. 17(10), pp. 2888 - 2899, 2016.

[7] B.-Y. Chen, W. H. K. Lam, Q. Li, A. Sumalee, K. Yan, "Shortest path finding problem in stochastic time-dependent road networks with stochastic first-in-first-out property", IEEE Trans. Intell. Transp. Syst., vol. 14, no. 4, pp. 1907-1917, Dec. 2013.

[8] M. Wooldridge, M. Jennings, «Intelligent agents: theory and practice". The Knowledge Engineering review 10(2), pp.115-152, 2002.

[9] B. Burmeister, A. Haddadi, and al, "Application of multi-agent in traffic and transportation". IEEE Proc. Software Engineering 144(1), pp. 51-60, 1997.

[10] Mc Graw-Hill, Science and technology, 2003.

[11] J. Cuena, J. Hernandez, and al, "Knowledge based models for adaptative traffic management systems", Transportation Research 3(5), pp. 311337, 1995.

[12] J. Hernandez, S. Ossowski, A. Garcia-Serrano, "Multiagent architectures for intelligent traffic management systems", Transportation Research Part C: Emerging Technologies, vol. 10, pp. 473-506, 2002.

[13] J. L. Adler, and V. J. Blue, "Cooperative multi-agent transportation management and route guidance systems", Transportation Research Part C, vol. 10, pp. 433-454, 2002.

[14] S. Ossowski, J. Z. Hernandez, and al, "Decision support for traffic management based on organizational and communicative multiagent abstraction”. Transportation Research Part C 13, pp. 272-298, 2005.

[15] M. Li, J. Hallam, and al, "A cooperative intelligent system for urban traffic problems". Proceedings of the 1996 IEEE International Symposium on intelligent Control, Dearborn, MI, pp.162-167, 1996.

[16] N. V. Finder, J. Strap, "A distributed approach to optimized control of street traffic signals". Journal of Transportation Engineering 118, pp. 99110, 1992.

[17] R. van Katwijk, P. van Koningsbruggen, "Coordination of traffic management instruments using agent technology". Transportation Research Part C: Emerging Technologies, vol. 10, Issues 5-6, pp. 455471, 2002.

[18] D. I. Robertson, "Research on the TRANSYT and SCOOT methods of signal coordination". ITE Journal, 1986.

[19] G. Scemama, "Développement d'un système à base de connaissance historique pour la gestion du trafic". Recherche Transport Sécurisé, R.T.S. 5(22), 1992.

[20] P. B. Hunt, D. L. Robertson, R. D. Bretherton, M. C. Royle, "The SCOOT online traffic signal optimization technique". Traffic Engineering and Control 23, pp. 190-199, 1982.

[21] Drogoul, A. Callinot, "Applying an Agent-Oriented Methodology to the Design of Artificial Organizations: A case study in Robotic Soccer". Journal of Autonomous Agents and multi-agent systems 1(1), pp. 113$129,1998$.

[22] R. L. Brooks, "On coloring the nodes of a network", Mathematical Proceedings of the Cambridge philosophical society, vol. 37, Issue 2, pp. 194-197, 1941

[23] M. Gondran, M. Minoux, "Graphes et algorithms". 1995.

[24] G. Abu-Lebdeh, A. K. Singh, "Modeling Arterial Travel Time with Limited Traffic Variables using Conditional Independence Graphs and State-Space Neural Networks". Procedia-Social and Behavioral Sciences, vol. 16, pp. 207-217, 2011. 